\title{
Erratum to: How habitat affects the benefits of communication in collectively foraging honey bees
}

\author{
Matina C. Donaldson-Matasci • Anna Dornhaus
}

Published online: 21 April 2012

(C) Springer-Verlag 2012

Erratum to: Behav Ecol Sociobiol

DOI 10.1007/s00265-011-1306-Z

The original version of this article unfortunately contained a mistake. The factor in the denominator of the third equation is missing.

Please see the correct equation below.

$\widehat{k}_{p}=\frac{\widehat{\lambda}_{f}}{\widehat{\lambda}_{p}}=\sqrt{\frac{\left(\sum_{i} k_{i}\right)\left(\sum_{i} l_{i}\right)\left(\sum_{i} \pi x_{i}^{2}\right)}{n^{2}\left(\frac{1}{2} \sum_{i} \pi y_{i}^{2}\right)}}$ 10.1007/s00265-011-1306-z.

M. C. Donaldson-Matasci $(\bowtie) \cdot$ A. Dornhaus

Department of Ecology and Evolutionary Biology,

University of Arizona,

Tucson, AZ 85721, USA

e-mail:matina@email.arizona.edu 